\title{
Performance of Templated Mesoporous Carbons in
}

\section{Supercapacitors}

\author{
M. Sevilla ${ }^{a}$, S. Álvarez ${ }^{a}$, T. A. Centeno ${ }^{a}$, A.B. Fuertes ${ }^{a}$ and F. Stoeckli \\ ${ }^{a}$ Instituto Nacional del Carbón-C.S.I.C., Apartado 73. 33080 Oviedo (Spain) \\ ${ }^{\mathrm{b}}$ IMT-Chimie des Surfaces, Université de Neuchâtel. Rue Emile Argand 11, CH-2009 \\ Neuchâtel, Switzerland
}

Abstract. By analogy with other types of carbons, templated mesoporous carbons (TMCs) can be used as supercapacitors. Their contribution arises essentially from the double layer capacity formed on their surface, which corresponds to $0.14 \mathrm{~F} \mathrm{~m}^{-2}$ in aqueous electrolytes such as $\mathrm{H}_{2} \mathrm{SO}_{4}$ and $\mathrm{KOH}$ and $0.06 \mathrm{~F} \mathrm{~m}^{-2}$ for the aprotic medium $\left(\mathrm{C}_{2} \mathrm{H}_{5}\right)_{4} \mathrm{NBF}_{4}$ in $\mathrm{CH}_{3} \mathrm{CN}$. In the case of a series of $27 \mathrm{TMCs}$, it appears that the effective surface area determined by independent techniques can be as high as 1500 to $1600 \mathrm{~m}^{2} \mathrm{~g}^{-1}$, and therefore exceeds the value obtained for many activated carbons (typically 900 to $1300 \mathrm{~m}^{2} \mathrm{~g}^{-1}$ ). On the other hand, the relatively low amount of surface oxygen in the present TMCs, as opposed to activated carbons, reduces the contribution of pseudo-capacitance effects and limits the gravimetric capacitance to 200-220 F g $\mathrm{g}^{-1}$ for aqueous electrolytes. In the case of non-aqueous electrolyte, it rarely exceeds $100 \mathrm{~F} \mathrm{~g}^{-1}$.

It is also shown that the average mesopore diameter of these TMCs does not improve significantly the ionic mobility compared with typical activated carbons of pore-widths above 1.0 to $1.3 \mathrm{~nm}$.

This study suggests that activated carbons remain the more promising candidates for supercapacitors with high performances.

\footnotetext{
* Corresponding autor. Tel.: +34.985119090; Fax: +34.985297662;

E-mail address: teresa@incar.csic.es (T.A. Centeno)
} 
Keywords: Mesoporous carbon; Template; Activated carbon; Surface areas; Capacitor. 


\section{Introduction}

Electrochemical capacitors [1] have generated wide interest in recent years for use in high power applications where high cycle efficiency and long cycle life are also required. One strategy for increasing the energy density stored in the electric double layer is the use of high surface area electrodes such as activated carbons (AC). These solids are essentially nanoporous, with locally slit-shaped cavities between 0.4 and $2.0 \mathrm{~nm}$ and relatively high internal surface areas, typically in the range of 700 to $1000 \mathrm{~m}^{2} \mathrm{~g}^{-1}$. Moreover, a careful selection of precursors and suitable activation protocols may lead to areas of approximately $1400 \mathrm{~m}^{2} \mathrm{~g}^{-1}[2,3]$.

It has been shown, that under the most favourable conditions, activated carbons can lead to gravimetric capacitances as high as $300 \mathrm{Fg}^{-1}$ for aqueous $\mathrm{H}_{2} \mathrm{SO}_{4}$ or $\mathrm{KOH}$ electrolytes. However, various studies [4-8] have pointed out the limitations of these materials for high power applications. For example, it has been reported that ionic motion may be hindered in narrow nanopores, which reduces the rate of energy delivery. This property, which is one of the advantages of electrical double layer capacitors (EDLCs), may therefore not be fully achieved. Furthermore, in the case of non-aqueous electrolytes such as $\left(\mathrm{C}_{2} \mathrm{H}_{5}\right)_{4} \mathrm{NBF}_{4}$ in $\mathrm{CH}_{3} \mathrm{CN}$, both charge storage and energy delivery rates can be limited by the size of the ions [5, 9-11]. It is therefore believed that high-surface-area templated carbons with a porosity formed almost exclusively by mesopores could be potentially more advantageous as EDLCs electrodes than activated carbons.

Research on the tailoring of the porous structure of active carbons for more demanding applications has been intensive in recent years. Thus, the classical activation procedures have been specifically adapted by using techniques such as the carbonization of polymer blends, doping the carbon precursors with heteroatoms, 
intercalation, and modified activation procedures [2, 12, 13]. However, a refined control of the pore size distribution and pore connectivity has not yet been achieved $[12,13]$.

Recently, the templating technique has been described in the literature as an alternative procedure for manufacturing porous carbons with well-defined structural characteristics (surface area, pore volume, pore size distribution, particle size, etc.) [12, 14-22]. Their synthesis is achieved by the filling of the pore system of an inorganic matrix with a carbon precursor and the subsequent carbonization of the composite. The inorganic template is finally removed by dissolving it in HF. Following this replication technique, porous carbons with narrow pore size distributions, almost exclusively in the mesopores range and with high specific surface areas, have been obtained from mesostructured silica materials [16-22].

Several studies have suggested promising properties of templated mesoporous carbons (TMC) when used in EDLC systems [23-33], but at the present stage the absence of a systematic analysis does not allow a reliable correlation between the structural characteristics of these materials and their capacitive behaviour. In fact, this approach is of prime importance for the design of carbon electrodes and electrochemical capacitors with improved performances.

The present study shows that a reliable characterization of the textural properties of templated mesoporous carbons allows a better understanding of their electrochemical ability for charge accumulation. Additionally, the possible technological and commercial advantages of TMC-based capacitors have been assessed by comparing them with classical activated carbons and it appears that the latter remain better candidates for supercapacitors. This is mainly due to the fact that activated carbons possess more surface functionalities than TMCs. 


\section{Experimental}

\subsection{Preparation of the templated mesoporous carbons}

A series of mesoporous carbons, widely differing in their porosity, was produced by inverse replication of mesoporous silica with tunable textural characteristics. Details about the preparation procedure and porous structure of the materials used as templates can be found elsewhere [17-21]. In a typical synthesis, mesoporous carbons were prepared by impregnating the pore system of the silica with paratoluenesulfonic acid $(0.5 \mathrm{M}$ in ethanol) as polymeric catalyst. Subsequently, furfuryl alcohol was added until incipient wetness, or until complete the pore volume of the silica The nanocomposites were cured in air at $80^{\circ} \mathrm{C}$ for 15 hours in order to induce the polymerization of furfuryl alcohol and the material was carbonised in nitrogen at $800^{\circ} \mathrm{C}$. The porous carbon itself was obtained by dissolving the silica framework in $48 \% \mathrm{HF}$ at room temperature. Finally, all carbons were heated for 2 hours at $1000^{\circ} \mathrm{C}$ in an inert atmosphere, in order to increase their electrical conductivity.

The samples of series CG (see Table 1) correspond to graphitized porous templated carbons. They were obtained by impregnating the freshly prepared carbons with a metallic salt and subjecting them to a further heat treatment at $900^{\circ} \mathrm{C}$ in nitrogen. Finally, the metallic particles were removed by washing the carbons with an acidic solution.

Carbons CGRF-1 and CGRF-11 were obtained with phenolic resin as carbon precursor. Their characteristics have already been described in a previous study [22].

2.2. Characterization of the textural and chemical properties of the carbons 
The main textural properties of the carbons have already been reported elsewhere [17-22].

In the present study, special care has been taken, in order to provide a reliable assessment of the surface area of templated mesoporous carbons. Therefore, different techniques were used $[3,33-35]$. The solids were characterized by the standard techniques based on $\mathrm{N}_{2}$ adsorption-desorption isotherms at $77 \mathrm{~K}$ (Micromeritics ASAP 2010) and immersion calorimetry at $293 \mathrm{~K}$ (Tian-Calvet-type calorimeter). The analysis of the nitrogen isotherms by different methods such as the BET equation, the comparison plot based on the reference nitrogen isotherm for Vulcan $3 G$ and the DFT method, assuming cylindrical pores, led to independent assessments of the surface of the carbons. This data was further cross-checked with the corresponding enthalpies of immersion of the carbons into benzene and into dilute aqueous solution of phenol $(0.4 \mathrm{M})$ [34]. The latter approach provides the total surface area on the basis of specific enthalpies of immersion of $-0.114 \mathrm{~J} \mathrm{~m}^{-2}$ for benzene and $-0.109 \mathrm{~J} \mathrm{~m}^{-2}$ for phenol adsorbed selectively from an aqueous solution onto the carbon surface, where it forms a monolayer. The amount of oxygen found on the surface of TMCs was obtained by a technique based on the measurement of the enthalpy of immersion of the carbons into water. The details of the experimental procedures can be found in the relevant references [3, 33-35].

\subsection{Analysis of the electrochemical properties of the carbons}

Capacitors were assembled in two-electrode Swagelock-type cells. The electrodes were prepared as composites by pressing a mixture of the active material (75 wt\%), polyvinylidene fluoride as binder (20 wt\%) and a carbon black (Super $\mathrm{P}$, $5 \mathrm{wt} \%)$. The preparation of the electrodes with TMCs required a particular care and, in 
some cases, the PVDF content was $25 \%$ to obtain handled items. Then, cylindrical pellets of $8 \mathrm{~mm}$ in diameter and $300-400 \mu \mathrm{m}$ in thickness were obtained. The positive and negative electrodes of comparable mass, ranging from 8 to $10 \mathrm{mg}$ were electrically isolated by a glassy fibrous separator. Stainless steel Hastelloy C-240 rods have been used as current collectors. Two aqueous media, $2 \mathrm{M} \mathrm{H}_{2} \mathrm{SO}_{4}$ and $6 \mathrm{M}$ $\mathrm{KOH}$, were used as electrolytes, as well as an aprotic $1 \mathrm{M}$ solution of $\left(\mathrm{C}_{2} \mathrm{H}_{5}\right)_{4} \mathrm{NBF}_{4}$ in $\mathrm{CH}_{3} \mathrm{CN}$ (The latter will be referred to as $\mathrm{TEABF}_{4} / \mathrm{AN}$ ). The electrochemical characterization was performed in an potentiostat/galvanostat Autolab-Ecochemie PGSTAT30 by cyclic voltammetry technique at scan rates from 1 to $50 \mathrm{mV} \mathrm{s}^{-1}$ and galvanostatic charge/discharge cycles at current densities between 1 and $100 \mathrm{~mA}$ $\mathrm{cm}^{-2}$. The voltage ranged from 0 to $0.8 \mathrm{~V}$ for aqueous electrolytes and between 0 and $2 \mathrm{~V}$ for organic medium. The specific capacitance, C, was expressed as Farad per gramm of carbon in the electrode.

\section{Results and discussion}

\subsection{Porosity of the templated mesoporous carbons}

A variety of templated carbons with narrow PSDs in the mesopore range were investigated (Table 1). As reported earlier [17-22], the size of the pores was selected by choosing the appropriate silica template. For the sake of clarity, the pore size distributions of typical samples are shown in Fig. 1. Carbons C-65-HT and C-50-120HT display narrow and unimodal pore size distributions with average pore diameters around $2.7 \mathrm{~nm}$ and $6.4 \mathrm{~nm}$, respectively. On the other hand, some TMCs display a second and wider pore system, consisting of mesopores between 5 and $30 \mathrm{~nm}$. For example, sample CZ53b-HT, also shown in Fig. 1, has a bimodal pore size distribution around with maxima near $3.2 \mathrm{~nm}$ and $14 \mathrm{~nm}$. 
The classical analysis of $\mathrm{N}_{2}$ adsorption isotherm following Dubinin's theory [3] provides information on the possible presence of micropores in the templated carbons. As described in detail elsewhere [3, 33, 35], values of Dubinin's characteristic energy $E_{o}$ below $15-16 \mathrm{~kJ} \mathrm{~mol}^{-1}$ suggest the absence of any significant microporosity in TMCs such as CZ16-HT, CZ34-HT, CZ71-HT and C-25-HT (Table 1). The domain of 15 to $16 \mathrm{~kJ} \mathrm{~mol}^{-1}$ itself corresponds to transition pores between supermicorpores and mesopores in which often no capillary condensation occurs. On the other hand, values of $E_{o}$ above $17 \mathrm{~kJ} \mathrm{~mol}^{-1}$ reflect the presence of micropores (The higher $E_{o}$, the smaller the micropore size $[3,35]$ ). It should be pointed out that the contribution of microporosity to the overall porosity is highest for the three carbons of series CG (around 45\%), with an average width around $1.4 \mathrm{~nm}\left(\mathrm{E}_{\mathrm{o}} \sim 19 \mathrm{~J}\right.$ $\left.\mathrm{mol}^{-1}\right)$.

It has been reported [35] that the specific surface areas estimated from the analysis of the nitrogen isotherms by the comparison plot $\left(\mathrm{S}_{\text {comp }}\right)$ and DFT $\left(\mathrm{S}_{\mathrm{DFT}}\right)$ were in good agreement with the values obtained from the technique outlined by Stoeckli et al. [3, 34, 35], based on the enthalpy of immersion into dilute aqueous solution of phenol ( $\left.S_{\text {phenol }}\right)$. Following an earlier study dealing with exclusively mesoporous carbons [33], where the enthalpy of immersion into benzene $\left(S_{\text {benzene }}\right)$ also provides information on the surface area itself, the average surface area obtained from the four different techniques, $S_{\text {total }}=\left(S_{\text {comp }}+S_{\text {phenol }}+S_{\text {benzene }}+S_{D F T}\right) / 4$, provides a reliable assessment of the surface area.

A first inspection of Table 1 shows that $1500-1600 \mathrm{~m}^{2} \mathrm{~g}^{-1}$ seems to be a realistic upper-bound for specific surface area of templated mesoporous carbons. However, as observed for activated carbons $[29,33,35]$, it appears that in the case of TMCs the BET-based surface area can also be much higher than the other four values. For 
example, for carbon CZ16-HT the average surface $S_{\text {total }}$ is $1561 \mathrm{~m}^{2} \mathrm{~g}^{-1}$, against 2610 $\mathrm{m}^{2} \mathrm{~g}^{-1}$ for $S_{\mathrm{BET}}$. The latter is obviously too high and may lead to erroneous specific properties related to this surface area (for example EDLCs expressed in $\mathrm{F} \mathrm{m}^{-2}$ ).

\subsection{Electrochemical properties of templated mesoporous carbons}

\subsubsection{Specific capacitance at low current density}

The capacitance of an EDLC electrode is expected to be proportional to the surface area of the solid, since it provides the interface for the electrochemical double layer. However, attempts to find a clear linear relationship between the gravimetric capacitance and surface areas, in particular $S_{\mathrm{BET}}$, have not been conclusive, as the specific capacitance $\left[\mathrm{Fm}^{-2}\right]$, may vary strongly from carbon to carbon $[5,36,37]$. This has been explained by different authors, who suggest that a large fraction of the carbon surface area is not accessible to the electrolyte ions and, consequently, the experimental capacitance $\left[\mathrm{Fg}^{-1}\right]$ of these materials depends strongly on the pore size distribution and the connectivity between the pores $[5,23-$ $25,31,32,36]$. In this context, many studies have been carried out to determine the optimum pore size distribution for the diffusion of the solvated ions within the carbon framework.

It has also been suggested that the capacitive behaviour of highly activated carbons is dominated by the electronic properties of the solid, rather than by the properties on the solution side of the double layer. For example, Kötz et al. [38, 39] ascribed the gravimetric capacitance limitation observed for active carbons with BETsurface areas above $1000 \mathrm{~m}^{2} \mathrm{~g}^{-1}$ to a space constriction for charge accomodation into the thin pore walls. 
As shown in Fig. 2, for templated carbons with surface areas of less than approximately $1000 \mathrm{~m}^{2} \mathrm{~g}^{-1}$ the electrical capacitance in $2 \mathrm{M} \mathrm{H}_{2} \mathrm{SO}_{4}$ at low current density $\left(\sim 1 \mathrm{~mA} \mathrm{~cm}^{-2}\right), \mathrm{C}_{\mathrm{o}}$, seems to increase linearly with both $\mathrm{S}_{\mathrm{BET}}$ and $\mathrm{S}_{\text {tot }}$, the two being similar. On the other hand, for higher BET surface areas, $\mathrm{C}_{\mathrm{o}}$ visibly levels off. The use of $\mathrm{S}_{\text {tot }}$ leads to an average value of 0.13 to $0.14 \mathrm{~F} \mathrm{~m}^{-2}$ for the aqueous $\mathrm{H}_{2} \mathrm{SO}_{4}$ and a similar value is suggested by a limited number of experiments with $\mathrm{KOH}$. These specific capacitances are similar to the values obtained for exclusively mesoporous carbons [33] and for non-porous carbons (graphite, graphitized carbon black, glassy carbons) [40]. It also appears that the contribution from the microporous surface of the templated carbons to $C_{0}$ does not differ from that of larger pores (meso- and macropores).

An overall assessment combining the data from the present work with data reported by other authors leads to the conclusion that the specific capacitance for TMCs is generally in the range $130-180 \mathrm{Fg}^{-1}$ in aqueous electrolytes. On the other hand, 200-220 $\mathrm{Fg}^{-1}$ seems to be the upper limit for this type of electrolyte [24-33]. This limit is in agreement with the expected value based on $1500-1600 \mathrm{~m}^{2} \mathrm{~g}^{-1}$ as a realistic upper-bound for the specific surface area of templated mesoporous carbons (see section 3.1).

TMCs have higher specific surface areas than many activated carbons and therefore, they may be potentially interesting candidates for use in supercapacitors. However, as seen in Figs. $3 a$ and $3 b$ based on our data and on data from the literature $[25,41,42,44]$, the specific capacitance in the aqueous $\mathrm{H}_{2} \mathrm{SO}_{4}$ and $\mathrm{KOH}$ electrolytes is significantly higher for many activated carbons having lower specific surface areas. As shown recently [41-43], their better performance is due to the fact that certain oxygen-containing surface complexes also contribute to $C_{0}$ in the form of 
a pseudo-capacitance, to be added to the purely double layer capacitance associated with the surface area [1,5,41-43]. The groups responsible for this contribution are those which desorb as CO in TPD (Thermally Programmed Desorption) and correspond to exposed edges or indentations in the carbon structure. For the $\mathrm{H}_{2} \mathrm{SO}_{4}$ electrolyte, their contribution is approximately $63 \mathrm{~F}$ per meq [CO], whereas the contribution of the basal plane to the EDLC is approximately 0.080 $\mathrm{F} \mathrm{m}^{-2}$. Assuming a surface area of $50 \mathrm{~m}^{2}$ per meq of $\mathrm{CO}$, which corresponds to $1.3 \mathrm{~F}$ $\mathrm{m}^{-2}$, it appears that the capacitance of $0.140 \mathrm{~F} \mathrm{~m}^{-2}$ observed for templated mesoporous carbons corresponds to $5 \%$ of the surface, occupied by these groups. It follows that the higher values of $\mathrm{C}_{0}$ (up to $0.250-0.260 \mathrm{~F} \mathrm{~m}^{-2}$ ) obtained for certain activated carbons, reflect the higher proportion of accessible edges in these carbons, as opposed to templated or non porous carbons. In the case of PX-21, a $\mathrm{KOH}$ activated carbon with a total oxygen content of $9.4 \mathrm{mmol} \mathrm{g}^{-1}$ and $8 \mathrm{mmol} \mathrm{g}^{-1}$ of surface oxygen, the groups contributing to $C_{0}$ occupy approximately $13 \%$ of the total surface area [41].

As shown in Fig. 3b, based on our data, as well as isolated data reported in the literature $[25,41,42,44]$, a pattern similar to that of $\mathrm{H}_{2} \mathrm{SO}_{4}$ applies to the $6 \mathrm{M} \mathrm{KOH}$ electrolyte.

The foregoing results indicate that the templated mesoporous carbons investigated in this study behave in aqueous media essentially as double-layer capacitors, due to their smaller density of surface functionalities. As a consequence, and in view of the relatively large contribution of CO-desorbing groups to the total capacitance (approximately 60 to $65 \mathrm{~F}$ per meq of $\mathrm{CO}$ ), the larger surface area of TMCs is not sufficient to compete with activated carbons.

Different authors have recently suggested the use precursors containing 
functional groups, in order to increase the specific capacitance of templated carbons. For example, Kodama et al. [28] and Frackowiak et al. [45] reported higher capacitances for carbons containing residual nitrogen from the precursor. Increased performances have also been reported for sucrose-based TMCs [25-27], obviously due to the higher oxygen content of the precursor.

In the case of a non-aqueous electrolyte such as $1 \mathrm{M} T E A B F_{4} / A N$, the series of TMCs shown in Table 1 leads to specific gravimetric capacitances which are significantly lower than those obtained in aqueous media (They rarely exceed $95 \mathrm{~F} \mathrm{~g}^{-}$ ${ }^{1}$ ). As illustrated in Fig. 4, the specific capacitance $C_{0}$ also increases linearly with the specific surface area but, in this case, $S_{\text {total }}$ leads to approximately $0.06 \mathrm{~F} \mathrm{~m}^{-2}$ as opposed to $0.14 \mathrm{~F} \mathrm{~m}^{-2}$ for the aqueous $\mathrm{H}_{2} \mathrm{SO}_{4}$ and $\mathrm{KOH}$ electrolytes. This ratio is in agreement with data reported by other authors [25-27] for TMCs with pore sizes around 3-4 nm and BET surface areas up to $1300 \mathrm{~m}^{2} \mathrm{~g}^{-1}$. Although these areas may be questioned, a good fit to the general pattern is observed.

As seen in Fig. 4, the specific capacitances of activated carbons in the aprotic electrolyte tend to be higher than the capacitances of the TMCs with similar surface areas. This may again indicate a higher pseudo-capacitance effect, rather than hindered accessibility in the mesopores. The latter may be ruled out, since experiments based on immersion calorimetry indicate that pores wider than approximately $1 \mathrm{~nm}$ are accessible to the electrolyte. The effect of other factors on the EDLC properties should be considered, such as such as affinity to electrolytic solution, wettability, conducting properties, and intrinsic properties of electrodes themselves.

Finally, a systematic analysis based on $\mathrm{H}_{2} \mathrm{SO}_{4}, \mathrm{KOH}$ and $\mathrm{TEABF}_{4} / \mathrm{AN}$ electrolytes, suggests that for low current densities, the capacitance does not depend significantly 
on the average pore diameters of the mesoporous carbons. The analysis of data found in the literature $[25-27,29]$ shows that the same pattern applies to other templated carbons and no improvement is detected for carbons with larger pore sizes (e.g. carbon C65b-HT, with a pore size around $8 \mathrm{~nm}$ ) or having bimodal pore size distributions (e.g. carbon CZ71-HT).

\subsubsection{Evolution of electrical capacitance with current density}

Recently, some authors have emphasized the ability of new advanced mesoporous carbons to store large amounts of energy with faster charge propagation than that achieved by microporous carbons [23-32]. It has been reported that the former suit the size of the electrolyte ions and enhance the EDLC performance at high rate.

Figure $5 a$ illustrates the evolution of normalised specific capacitance $\left(C / C_{0}\right)$ with current density $d$ for TMCs and for activated carbons in $2 \mathrm{M} \mathrm{H}_{2} \mathrm{SO}_{4}$ electrolyte. The slight relative decrease in $\mathrm{C} / \mathrm{C}_{\mathrm{o}}$ for templated mesoporous carbons (around $15 \%$ at $10 \mathrm{~mA} \mathrm{~cm}^{-2}$ and $30 \%$ at $100 \mathrm{~mA} \mathrm{~cm}{ }^{-2}$ ) confirms that the ion mobility is facilitated and the formation of an effective double-layer occurs even at high current density.

The comparison of the performance of TMCs with unimodal pore size distributions centered at $2.3 \mathrm{~nm}$ (C-50-HT), $3.9 \mathrm{~nm}(\mathrm{C}-50-100-\mathrm{HT}), 6.4 \mathrm{~nm}(\mathrm{C}-50-120-\mathrm{HT})$ and 8.0 $\mathrm{nm}(\mathrm{C} 65 \mathrm{~b}-\mathrm{HT})$ reveals no significant influence of the pore dimensions on the evolution of the specific capacitance with current density. Furthermore, the enhancement of the accessibility reported for bimodal pore size distributions [46], is not confirmed by the present study. This is illustrated by TMC C82-HT, where the additional system of larger pores has no influence on the general pattern. 
The profiles shown in Fig. 5a may also lead to the conclusion that TMCs with a narrow pore size distribution around 2-3 nm optimize the supercapacitor performance in aqueous media. However, the detailed analysis of the performance of microporous activated carbons with average micropore size of $1.1 \mathrm{~nm}$ (BV-46), 1.5 $\mathrm{nm}$ (Super DLC-50) and $2.1 \mathrm{~nm}(\mathrm{~N}-125)$ shows that the diffusion of aqueous ions is not strongly hindered in this type of microporous network. In fact, their capacitive behaviour at high current density even appears to be somewhat better than that observed for mesoporous carbons.

As shown elsewhere [37, 41, 42], for porous carbons obtained by strong activation, the motion of ionic species depends, to some extent, on the pore width but the large amount of $\mathrm{CO}_{2}$-generating groups in TPD has a predominant effect on the discharge rate of the devices. For example, this is the case for the $\mathrm{KOH}$-activated carbon PX-21, which has an average micropore size around $2.0 \mathrm{~nm}$ and an oxygen content of $8 \mathrm{mmol} \mathrm{g}^{-1}$. As the current density increases, the capacitance of PX-21 drops whereas the active carbon $\mathrm{N}-125$ with a similar micropore width $(2.1 \mathrm{~nm})$ but only $0.22 \mathrm{mmol} \mathrm{g}^{-1}$ of oxygen, retains about $80 \%$ of their initial capacitance even at $100 \mathrm{~mA} \mathrm{~cm}{ }^{-2}$. The same applies for activated carbons BV46 and Super DLC-50 with average micropore sizes of 1.1 and 1.5 and oxygen contents of respectively 0.32 and $0.98 \mathrm{mmol} \mathrm{g}^{-1}$.

A closer examination of the data suggests that the better performance at high current density reported for templated mesoporous carbons compared to some highly activated carbons should not be ascribed exclusively to differences in structural properties. The lower total oxygen content found on the surface of the TMCs obtained by carbonization alone $\left(\sim 1.5 \mathrm{mmol} \mathrm{g}^{-1}\right)$ probably explains their good behaviour in aqueous electrolytes at high current densities. 
Figure $5 \mathrm{~b}$ suggests a more complex behaviour in the case of the aprotic electrolyte $\mathrm{TEABF}_{4} / \mathrm{AN}$. The slight decrease in $\mathrm{C} / \mathrm{C}_{0}$ at $100 \mathrm{~mA} \mathrm{~cm}{ }^{-2}$ observed for TMCs with a pore size around 2-3 nm (C-25-HT and CZ53b-HT) suggests very promising rate capability in this electrolyte. However, decreases larger than $30 \%$ for samples with wider pores, such as $\mathrm{CZ} 35-\mathrm{HT}$ and $\mathrm{C} 82-\mathrm{HT}$, clearly indicate that other factors must be considered beside the pore size distribution and/or the oxygen content. The latter may be neglected as far as all TMCs were prepared from the same precursor at $800^{\circ} \mathrm{C}$ and have the same low oxygen content.

According to different authors, factors such as the length and the connectivity of the pores may play a role in the ions mobility during charging/discharging processes $[5,47]$. Furthermore, the size and/or the morphology of the particles may affect the TMCs/binder agglomeration in the aprotic medium and, therefore have an influence on the properties of the TMCs-electrodes.

\subsubsection{Dependence between power output and energy density for TMC capacitors}

Ragone-type plots, which relate power-density to achievable energy-density, have been used for comparative evaluation of the different carbons investigated in this study. The thickness and the mass of the electrodes were determined individually, and no significant change in the thickness of carbon electrode was observed for carbons with different specific surface areas. This thickness was around $300 \mu \mathrm{m}$ for all electrodes. It follows that similar profiles (although with a scaling factor) were obtained by using either unit mass or unit volume.

The data was obtained with laboratory scale devices and it is likely that the performance would be improved by using a commercial set up. Obviously, there may be numerical changes, depending on the capacitor configuration, but the relative 
performances of the carbons can be derived from their behaviour under the same experimental conditions.

As illustrated by Fig. 6, Ragone-type plots display the usual 'hooked' shape corresponding to a fall-off of energy-density as the power drain is increased in the discharge of the capacitor. A specific energy between 3 and $5 \mathrm{Wh} \mathrm{kg}^{-1}$ of active mass of templated mesoporous carbons is obtained with a $0.8 \mathrm{~V}$ aqueous capacitor. The use of nonaqueous electrolyte is preferred since the stored energy increases with the square of the operating voltage. Thus, one reaches values around $12-14 \mathrm{Wh} \mathrm{kg}^{-1}$ for 2 Volts $\mathrm{TEABF}_{4} / \mathrm{AN}$ capacitors. Although part of this increase is compensated by the higher resistance of the aprotic electrolyte [1,5], TMC-capacitors provide about 3.5 Wh kg ${ }^{-1}$ at power in the 1200 and $5700 \mathrm{~W} \mathrm{~kg}^{-1}$ range, whereas $2 \mathrm{M} \mathrm{H}_{2} \mathrm{SO}_{4}$ devices achieve power densities between 700 and $2500 \mathrm{~W} \mathrm{~kg}^{-1}$ at $1 \mathrm{Wh} \mathrm{kg}^{-1}$.

It must be pointed out that no clear correlation between the release of the stored energy and the pore size of the TMCs has been found yet. For example, Fig. 6 indicates a poor capacitor performance for carbons with large pore size, such as C65b-HT in $2 \mathrm{M} \mathrm{H}_{2} \mathrm{SO}_{4}$ or $\mathrm{C} 82-\mathrm{HT}$ in $1 \mathrm{M} \mathrm{TEABF} / \mathrm{AN}$. Furthermore, the expected advantages for TMCs over ACs as electrodes in electrochemical capacitors are not obvious and the device corresponding to microporous carbons Super DLC-30 and M30, appear to have a similar reliability at high power to some TMCs.

From the foregoing it follows that the physico-chemical characteristics of carbons are not the only factors controlling the release of the stored electric energy and other properties such as the affinity for the electrolytic solution, wettability, conducting properties, device configuration and electrode processing should also be considered. 


\section{Conclusions}

The systematic analysis of the supercapacitor performance of a variety of templated mesoporous carbons leads to the conclusion that

(a) These materials behave mainly as electrical double-layer capacitors through the extent of their total surface area. The average specific capacitances are around $0.130-0.140 \mathrm{~F} \mathrm{~m}^{-2}$ for $2 \mathrm{M} \mathrm{H}_{2} \mathrm{SO}_{4}$ and $6 \mathrm{M} \mathrm{KOH}$ electrolytes and $0.06 \mathrm{~F} \mathrm{~m}^{-2}$ for $1 \mathrm{M} \mathrm{TEABF} / \mathrm{AN}$.

(b) For the present TMCs, based on the same carbon precursor, the pseudocapacitive contribution is negligible due to the low content of oxygen-containing surface groups.

(c) It also appears that the contribution of the microporous surface of TMCs, if present at all, to the electrical capacitance does not differ from that of larger pores (meso- and macropores).

(d) An overall assessment of the total surface area combining the data from $\mathrm{N}_{2}$ adsorption and immersion calorimetry indicates that $1500-1600 \mathrm{~m}^{2} \mathrm{~g}^{-1}$ is a realistic upper-bound for templated mesoporous carbons. This means that the maximum gravimetric capacitance for most TMCs is limited to $200-220$ $\mathrm{F} \mathrm{g}^{-1}$ in aqueous electrolytes and in the case of a non-aqueous electrolyte such as $1 \mathrm{M} \mathrm{TEABF} 4 / \mathrm{AN}$, it would not exceed $100 \mathrm{Fg}^{-1}$.

(e) A systematic analysis based on aqueous $2 \mathrm{M} \mathrm{H}_{2} \mathrm{SO}_{4}, 6 \mathrm{M} \mathrm{KOH}$ and $1 \mathrm{M}$ $\left(\mathrm{C}_{2} \mathrm{H}_{5}\right)_{4} \mathrm{NBF}_{4}$ in $\mathrm{CH}_{3} \mathrm{CN}$ electrolytes suggests that at low current densities the specific capacitance of mesoporous carbons does not depend significantly on the pore width itself and that no improvement is achieved for carbons with pore size above than 2-3 nm. 
(f) A closer examination suggests that the better performance reported for templated mesoporous carbons at high current density $d$ than for certain highly activated carbons, is not exclusively due to structural properties. It appears that the lower oxygen content $\left(\sim 1.5 \mathrm{mmol} \mathrm{g}^{-1}\right)$ of the TMCs obtained by carbonization alone, and consequently the lower proportion of acidic groups, explains the improved EDLCs-performance at high current density in aqueous electrolyte. This is an advantage over activated carbons, which contain more acidic groups.

(g) At this stage no clear correlation can be established between the release of the stored energy and the pore size of the present TMCs. It is likely that a number of other factors such as the affinity to electrolytic solution, wettability, conducting properties, device configuration, electrode processing must be considered in further studies.

(h) From a general point of view, the expected advantages for TMCs over ACs as electrodes in electrochemical capacitors are not obvious.

\section{Acknowledgements}

The financial support provided by the Spanish MCyT (MAT2005-00262) is gratefully acknowledged. M. Sevilla and S. Alvarez thank the Spanish MCyT for their respective FPU (AP-2004-0027) and FPI (BES-2003-0134) grants.

\section{References}

[1] B.E.Conway, Electrochemical Supercapacitors. New York; Kluwer Academic, 1999, 105-124. 
[2] F. Rodríguez-Reinoso, in: F. Schüth, K.S.W. Sing, J. Weitkamp (Eds.), Handbook of Porous Solids, vol. 3, Wiley-VCH, Weinheim, 2002, 1766-1827.

[3] F. Stoeckli, In: J. Patrick (Ed.), Porosity in carbons-characterization and applications, Arnold, London, 1995, 67-97.

[4] R. Kötz, M. Carlen, Electrochim. Acta 45 (2000) 2483.

[5] E. Frackowiak, F. Béguin, Carbon 39 (2001) 937.

[6] C. Emmenegger, P. Mauron, P. Sudan, P. Wenger, V. Hermann, R. Gallay, A. Züttel, J. Power Sources 124 (2003) 321.

[7] Y.J. Kim, Y. Horie, Y. Matsuzawa, S. Ozaki, M. Endo, M.S. Dresselhaus, Carbon 42 (2004) 2423.

[8] J. Niu, W.G. Pell, B.E. Conway, J. Power Sources 156 (2006) 725.

[9] M. Endo, T. Maeda, T. Takeda, Y.J. Kim, K. Koshiba, H. Hara, M.S. Dresselhaus, J. Electrochem. Soc. 148 (2001) A910.

[10] B. Kastening, M. Heins, Electrochim. Acta 50 (2005) 2487.

[11] M. Arulepp, L. Permann, J. Leis, A. Perkson, K. Rumma, A. Jänes A, E. Lust, J. Power Sources 133 (2004): 320.

[12] T. Kyotani, Carbon 38 (2000) 269.

[13] M. Inagaki, New Carbons-control of structure and functions, Elsevier, Amsterdam 2000, 124-34.

[14] R. Ryoo, S.H. Joo, M. Kruk, M. Jaroniec, Adv. Mater. 13 (2001) 677.

[15] B. Sakintuna, Y. Yürüm, Ind. Eng. Chem. Res. 44 (2005) 2893.

[16] J. Lee, S. Han, T. Hyeon, J. Mater. Chem. 14 (2004) 478.

[17] A.B. Fuertes, D.M. Nevskaia, Micropor. Mesopor. Mater. 62 (2003) 177.

[18] A.B. Fuertes, J. Mater. Chem. 13(2003) 3085.

[19] S. Alvarez, A.B. Fuertes, Carbon 42 (2004) 433 
[20] A.B. Fuertes, Chem. Mater. 16 (2004) 449

[21] M. Sevilla, S. Alvarez, A.B. Fuertes, Micropor. Mesopor. Mater. 74 (2004) 49.

[22] M. Sevilla, A.B. Fuertes, Carbon 44 (2006) 468.

[23] S. Yoon, J. Lee, T. Hyeon, S.M. Oh, J. Electrochem. Soc. 147 (2000) 2507.

[24] H. Zhou, S. Zhu, M. Hibino, I. Honma, J. Power Sources 122 (2003): 219.

[25] K. Jurewicz, C. Vix-Guterl, E. Frackowiak, S. Saadallah, M. Reda, J. Parmentier, J. Patarin, F. Béguin, J. Phys. Chem. Solids 65 (2004) 287.

[26] C. Vix-Guterl, S. Saadallah, K. Jurewicz, E. Frackowiak, M. Reda, J. Parmentier , J. Patarin, F. Beguin, Mat. Sci. Eng. B 108 (2004) 148.

[27] C. Vix-Guterl, E. Frackowiak, K. Jurewicz, M. Friebe, J. Parmentier, F. Béguin, Carbon 43 (2005) 1293.

[28] M. Kodama, J. Yamashita, Y. Soneda, H. Hatori, S. Nishimura, K. Kamegawa, Mat. Sci. Eng. B 108 (2004) 156.

[29] H.Y. Liu, K.P. Wang, H. Teng, Carbon 43 (2005) 559.

[30] W. Xing, S.Z. Qiao, R.G. Ding, F. Li, G.Q. Lu, Z.F. Yan, H.M. Cheng, Carbon 44 (2006) 216.

[31] L. Li, H. Song, X. Chen, Electrochim. Acta 2006 (in press)

[32] L. Zhou, H. Li, C. Yu, X. Zhou, J. Tang, Y. Meng, Y. Xia, D. Zhao, Carbon 44 (2006) 1581.

[33] T.A. Centeno, M. Sevilla, A.B. Fuertes, F. Stoeckli, Carbon 43 (2005) 3012.

[34] F. Stoeckli, M.V. López-Ramón, C. Moreno-Castilla, Langmuir 17 (2001) 3301.

[35] F. Stoeckli, T.A. Centeno, Carbon 43 (2005) 1184.

[36] K. Kierzek, E. Frackowiak, G. Lota, G. Gryglewicz, J. Machnikowski, Electrochim. Acta 49 (2004) 515.

[37] T.A. Centeno, F. Stoeckli, J. Power Sources 154 (2006) 314. 
[38] F. Raimondi, G.G. Scherer, R. Kötz, A. Wokaun, Angw. Chem. Int. Ed. 44 (2005) 2190.

[39] O. Barbieri, M. Hahn, A. Herzog, R. Kötz, Carbon 43 (2005) 1303.

[40] K. Kinoshita, Carbon Electrochemical and Physicochemical Properties, John Wiley, New York, 1988, 293-295.

[41] T.A. Centeno, F. Stoeckli, Electrochim. Acta 2006 (in press)

[42] T.A. Centeno, F. Stoeckli, in: V. Gupta (Ed.), Recent Advances in Supercapacitors, Transworld Research Network, Kerala, 2006, 57-77.

[43] M.J. Bleda-Martínez, J.A. Maciá-Agulló, D. Lozano-Castelló, E. Murallón, D. Cazorla-Amorós, A. Linares-Solano, Carbon 43 (2005) 2677.

[44] E. Raymundo-Piñero, K. Kierzek, J. Machnikowski, F. Béguin, Carbon 44 (2006) 2498

[45] E. Frackowiak, G. Lota, J. Machnikowski, C. Vix-Guterl, F. Béguin, Electrochim. Acta 51 (2006) 2209.

[46] A.B. Fuertes, F. Picó, J.M. Rojo, J. Power Sources 133 (2004) 329.

[47] G.J. Lee, S.I. Pyun, C.H. Kim, J. Solid State Electrochem. 8 (2004) 110. 
Figure 1. Pore size distributions for templated mesoporous carbons C-65-HT (口), CZ53b-HT (x) and C-50-120-HT (•)

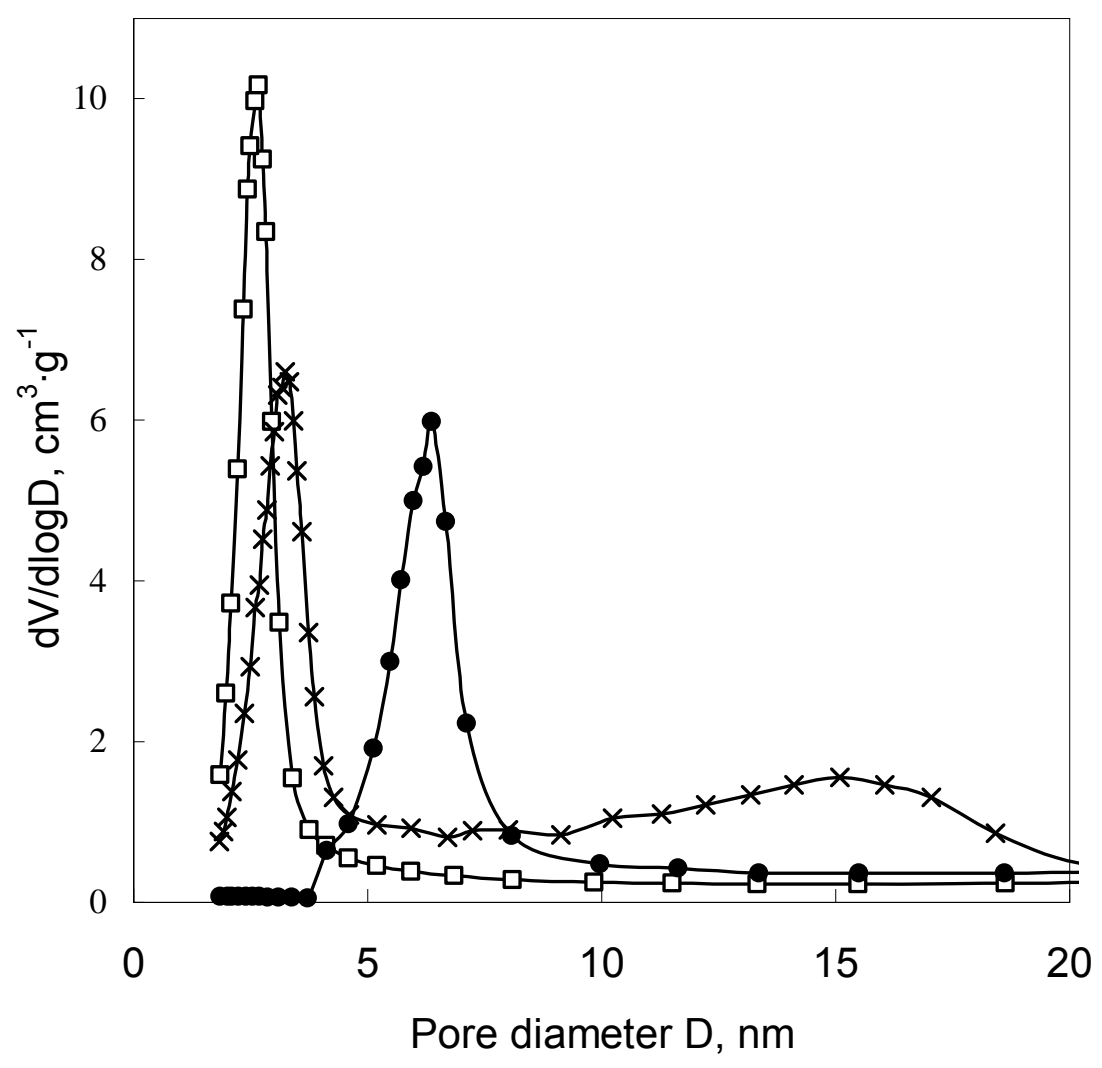


Figure 2. Variation of the specific capacitance $\left(1 \mathrm{~mA} . \mathrm{cm}^{-2}\right)$ of templated mesoporous carbons with the total surface area ( $\mathbf{m})$ and the BET-surface area (o) in $2 \mathrm{M} \mathrm{H}_{2} \mathrm{SO}_{4}$.

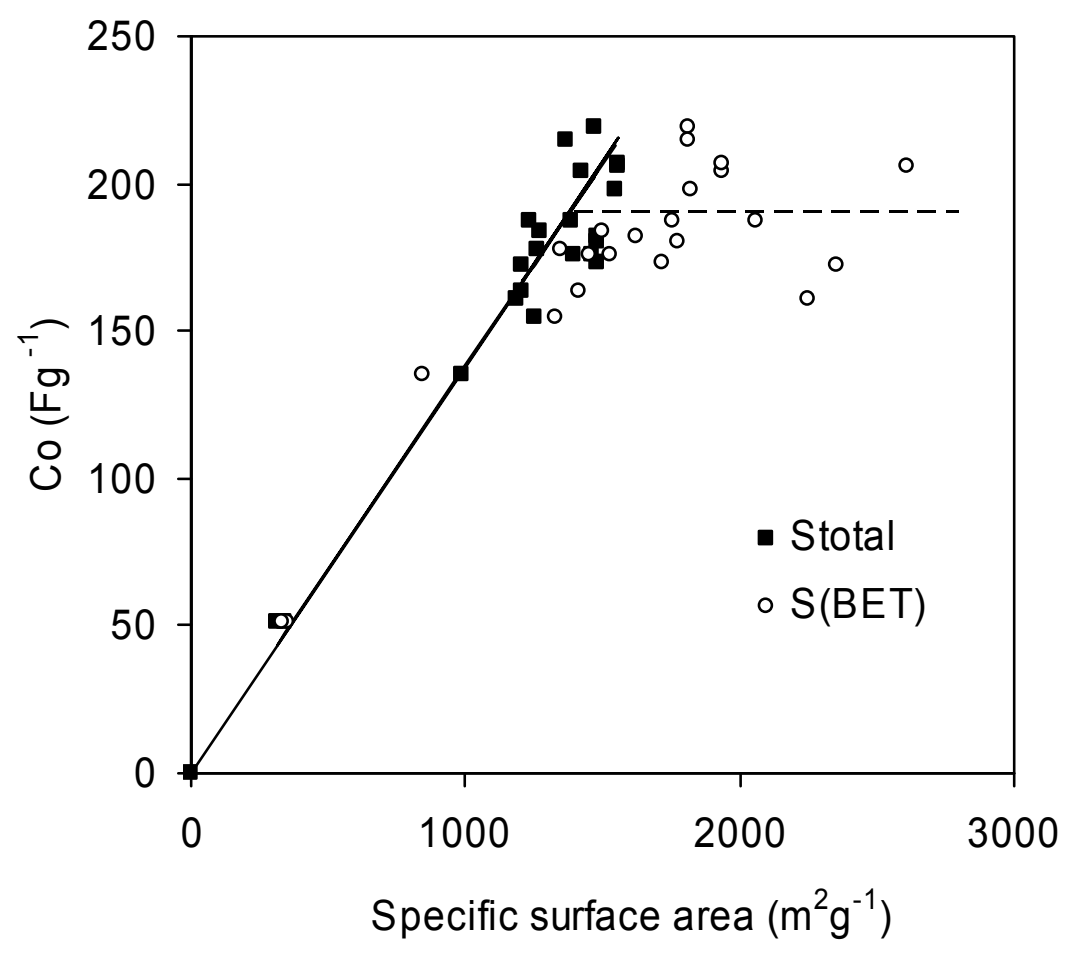


Figure 3. Variation of the specific capacitance $\left(1 \mathrm{~mA} \mathrm{~cm}^{-2}\right)$ in $2 \mathrm{M} \mathrm{H}_{2} \mathrm{SO}_{4}$ (a) and $6 \mathrm{M}$ $\mathrm{KOH}$ (b) electrolytes with the total surface area for templated mesoporous carbons of Table 1 ( $\mathbf{)}$ ) and from ref. [25] ( $\mathbf{\Lambda}$ ) and activated carbons from refs. [41, 42] ( $\square$ ) and [44] (०). The line through the origin corresponds to a linear best fit for the TMCs.
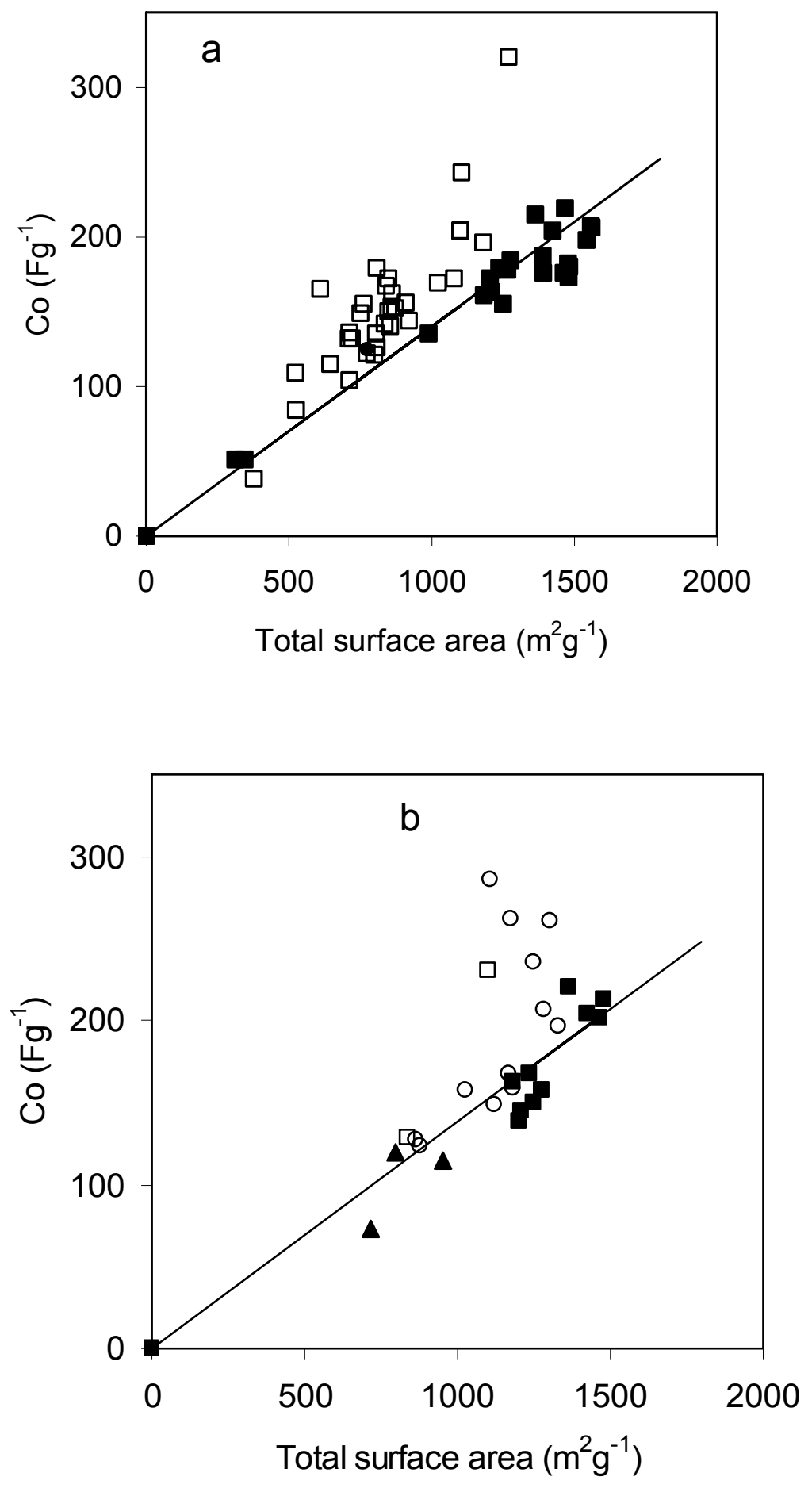
Figure 4. Variation of the specific capacitance $\left(1 \mathrm{~mA} \mathrm{~cm}^{-2}\right)$ in $1 \mathrm{M} \mathrm{TEABF} / \mathrm{AN}$ electrolyte with the total surface area for templated mesoporous carbons of Table 1 (-) and from refs. [25-27] ( $\mathbf{A}$ ) and activated carbons from refs. [39] (0) and [41, 42] ( $\square$ ).

The line through the origin corresponds to a linear best fit for the TMCs.

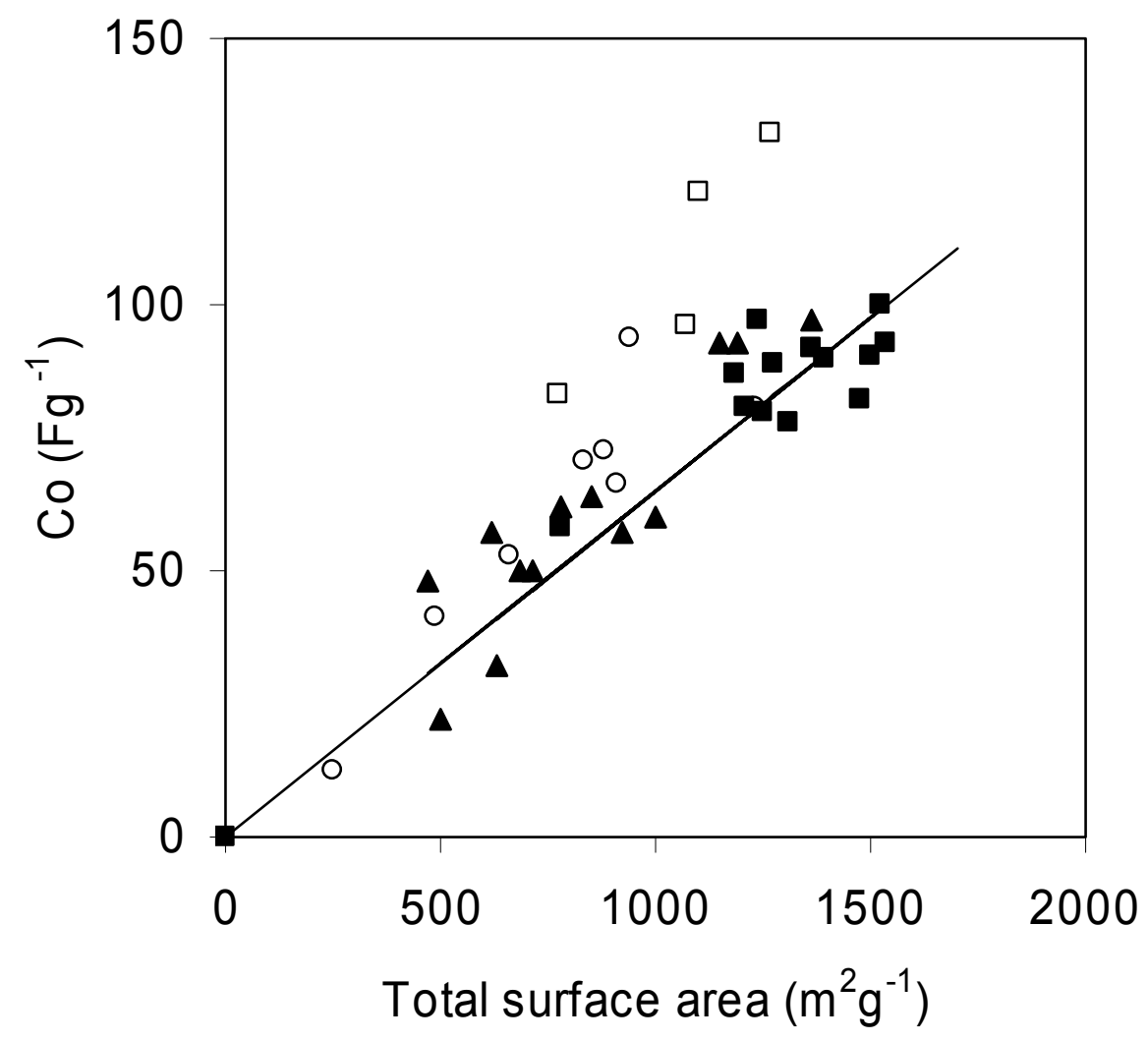


Figure 5. Decrease of the normalized specific capacitance $(\mathrm{C} / \mathrm{Co})$ with increasing current density $d$ in $2 \mathrm{M} \mathrm{H}_{2} \mathrm{SO}_{4}(\mathrm{a})$ and $1 \mathrm{M} \mathrm{TEABF}_{4} / \mathrm{AN}(\mathrm{b})$. The average pore sizes of the carbons are given in brackets

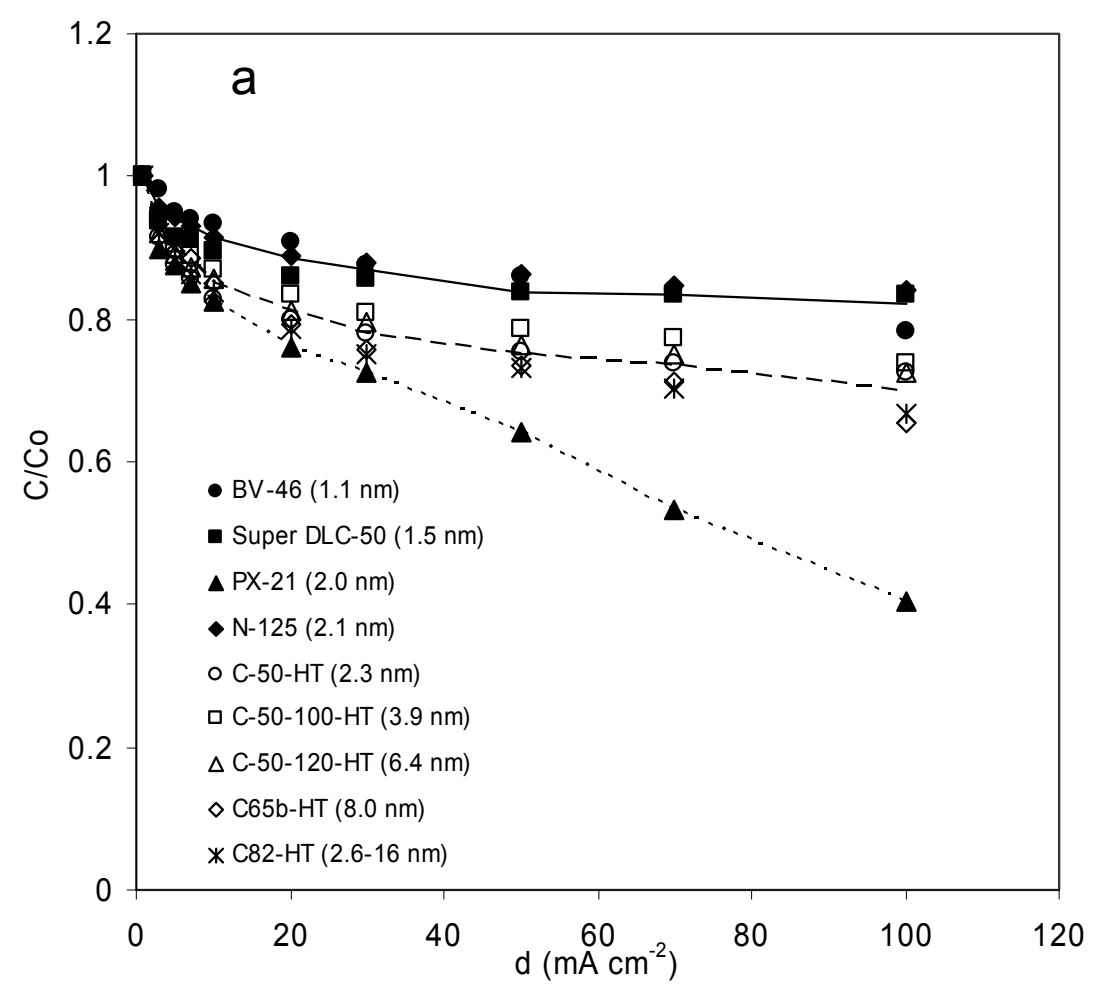




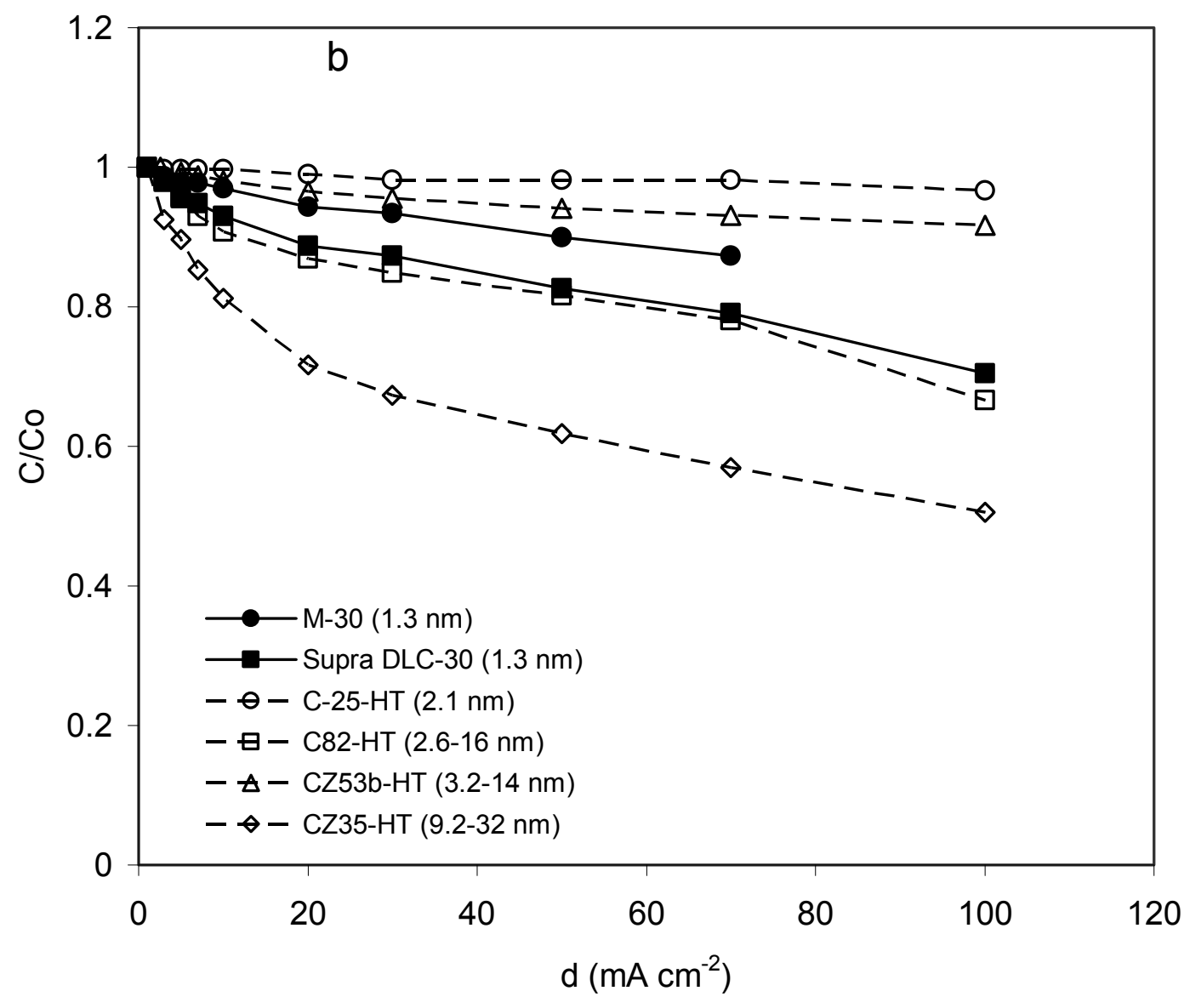


Figure 6. Ragone-type plots for different carbons: Active carbons : M-30 (+), Super DLC-30 (x) and Templated mesoporous carbons: C-25-HT [2.1 nm] (०), C-65-HT [2.7

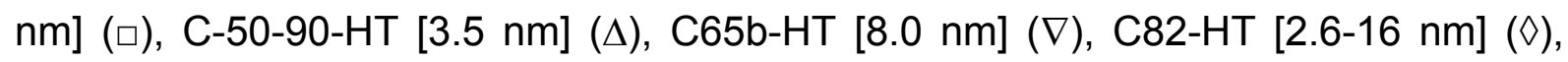
CZ35-HT [9.2-32 nm] (区)

Open symbols for $\mathrm{H}_{2} \mathrm{SO}_{4}$ electrolyte

Closed symbols for organic electrolyte.

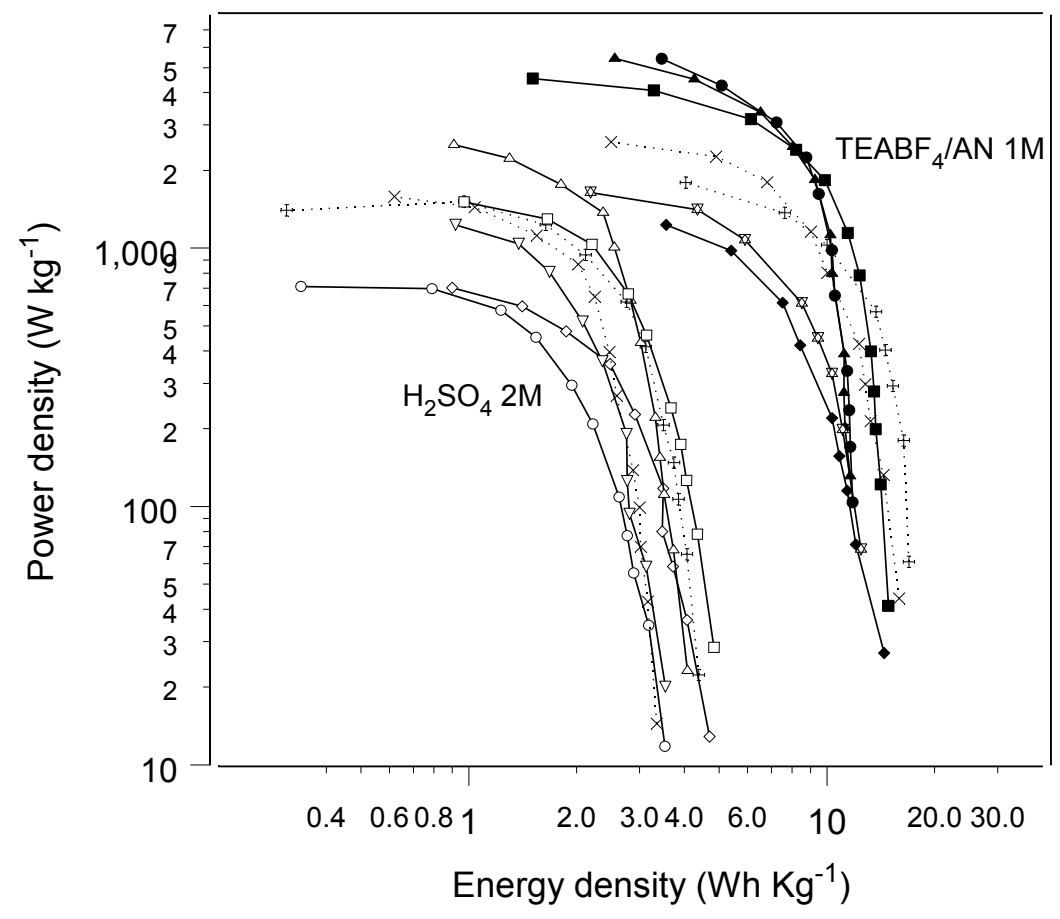


Table 1. Structural characteristics of the templated carbons.

\begin{tabular}{|c|c|c|c|c|c|c|c|c|c|c|}
\hline $\begin{array}{c}\text { Templated } \\
\text { Carbon }\end{array}$ & $\begin{array}{c}\text { Silica } \\
\text { Template }\end{array}$ & $\begin{array}{c}V_{p} \\
\left(\mathrm{~cm}^{3} \mathrm{~g}^{-1}\right)\end{array}$ & $\begin{array}{c}\mathrm{D}_{\mathrm{KJS}}{ }^{\mathrm{a}} \\
(\mathrm{nm})\end{array}$ & $\begin{array}{c}\mathrm{E}_{\mathrm{o}} \\
\left(\mathrm{kJ} \mathrm{mol}^{-1}\right)\end{array}$ & $\begin{array}{l}S_{\text {total }} b \\
\left(\mathrm{~m}^{2} \mathrm{~g}^{-1}\right)\end{array}$ & $\begin{array}{c}S_{B E T} \\
\left(m^{2} g^{-1}\right)\end{array}$ & $\begin{array}{c}\text { Pore } \\
\text { Structure }\end{array}$ & $\begin{array}{c}\text { Particle } \\
\text { morphology }\end{array}$ & $\begin{array}{c}\text { Particle size } \\
(\mu \mathrm{m})\end{array}$ & Ref. \\
\hline C60-HT & SBA-12 & 1.07 & 2.4 & 16.7 & 1468 & 1810 & Cubic 3D ordered & Spherical & $2-8$ & {$[18]$} \\
\hline CE8-HT & SBA-16 & 1.19 & 3.5 & 16.4 & 1544 & 1820 & 3D, low order & Spherical & $2-8$ & {$[18]$} \\
\hline $\mathrm{C} 65 \mathrm{~b}-\mathrm{HT}$ & SBA-16 & 1.95 & 8.0 & 16.8 & 1536 & 1510 & 3D,low order & Spherical & $2-8$ & {$[17]$} \\
\hline $\mathrm{C} 82-\mathrm{HT}$ & SBA-11 & 1.74 & $2.6+16$ & 16.8 & 1364 & 1810 & 3D disordered & Irregular & $2-8$ & {$[17]$} \\
\hline CE1-HT & SBA-16 & 1.87 & $2.8+43$ & 17.1 & 1464 & 1530 & $3 \mathrm{D}$, low order & Agglomerates & $0.2-0.5$ & [18] \\
\hline $\mathrm{C} 91-\mathrm{HT}$ & SBA-16 & 1.65 & $3.5+24$ & 16.2 & 1478 & 1620 & 3D, low order & Agglomerates & $0.01-0.02$ & [18] \\
\hline CZ16-HT & MSU-1 & 1.75 & $2.7+7$ & 13.8 & 1561 & 2610 & wormhole & Spherical & $2-8$ & {$[19]$} \\
\hline CZ17-HT & MSU-1 & 1.33 & 2.9 & 16.2 & 1560 & 1930 & wormhole & Spherical & $2-8$ & [19] \\
\hline CZ34-HT & MSU-1 & 1.94 & 3.6 & 15.6 & 1425 & 1940 & wormhole & Spherical & $2-8$ & [19] \\
\hline $\mathrm{CZ53b-HT}$ & MSU-1 & 1.81 & $3.2+14$ & 16.9 & 1237 & 1760 & wormhole & Spherical & $2-8$ & [19] \\
\hline CZ42-HT & MSU-1 & 1.72 & $6.6+26$ & 17.1 & 1266 & 1350 & wormhole & Spherical & $2-8$ & [19] \\
\hline CZ35-HT & MSU-1 & 2.0 & $9.2+32$ & 16.6 & 1251 & 1340 & wormhole & Spherical & $2-8$ & {$[19]$} \\
\hline $\mathrm{CZ71-HT}$ & MSU-1 & 2.36 & $9.5+28$ & 15.4 & 1393 & 1450 & wormhole & Spherical & $2-8$ & [19] \\
\hline CW20-HT & MSU-1 & 1.68 & 3.5 & 17.5 & 1305 & 1310 & wormhole & Agglomerates & $0.1-0.3$ & - \\
\hline CW16-HT & MSU-1 & 1.78 & 3.6 & 18.5 & 1215 & 1230 & wormhole & Agglomerates & $0.1-0.3$ & - \\
\hline C-25-HT & HMS & 1.91 & 2.1 & 13.7 & 1184 & 2250 & wormhole & Spherical & $0.07-0.25$ & {$[21]$} \\
\hline C-50-HT & HMS & 2.51 & 2.3 & 14.9 & 1206 & 2340 & wormhole & Spherical & $0.07-0.25$ & [21] \\
\hline C-65-HT & HMS & 2.09 & 2.7 & 14.7 & 1529 & 2060 & wormhole & Spherical & $0.07-0.25$ & [21] \\
\hline C-90-HT & HMS & 2.35 & 3.4 & 16.5 & 1480 & 1720 & wormhole & Spherical & $0.07-0.25$ & [21] \\
\hline C-50-90-HT & HMS & 1.87 & 3.5 & 13.6 & 1276 & 1500 & wormhole & Spherical & $0.07-0.25$ & [21] \\
\hline C-50-100-HT & HMS & 1.81 & 3.9 & 16.7 & 1209 & 1410 & wormhole & Spherical & $0.07-0.25$ & [21] \\
\hline C-50-120-HT & HMS & 1.83 & 6.4 & 16.9 & 1251 & 1330 & wormhole & Spherical & $0.07-0.25$ & [21] \\
\hline C-50-3/7-HT & HMS & 1.69 & $2.5+5.5$ & 14.2 & 1389 & 2060 & wormhole & Spherical & $0.07-0.25$ & [21] \\
\hline C-50-5/5-HT & HMS & 2.28 & $2.2+4.8$ & 15.7 & 1483 & 1780 & wormhole & Spherical & $0.07-0.25$ & [21] \\
\hline CG-1 & HMS & 0.61 & - & 19.02 & 346 & 356 & graphitic+amorphous & Agglomerates & - & - \\
\hline CGRF-1 & Xerogel & 0.35 & $2.8+6.9$ & 19.4 & 313 & 335 & Graphitic+amorphous & Agglomerates & - & {$[22]$} \\
\hline CGRF-11 & Xerogel & 0.77 & $3.4+13$ & 18.9 & 991 & 846 & Graphitic+amorphous & Agglomerates & - & [22] \\
\hline
\end{tabular}

${ }^{\mathrm{a}}$ Maximum/a of pore size distribution(s). (Diameter of cylindrical pores)

${ }^{\mathrm{b}} \mathrm{S}_{\text {total }}=\left(\mathrm{S}_{\text {comp }}+\mathrm{S}_{\text {phenol }}+\mathrm{S}_{\text {benzene }}+\mathrm{S}_{\mathrm{DFT}}\right) / 4$ 